\title{
Ações para o fortalecimento da resiliência em adolescentes
}

\author{
Actions for enhancing resilience among adolescents
}

Laís Katharina da Paixão dos Santos (https://orcid.org/0000-0002-0144-7109) ${ }^{1}$

Cláudia de Carvalho Santana (https://orcid.org/0000-0003-1828-5529) ${ }^{2}$

Marta Vanessa Oliveira de Souza (https://orcid.org/0000-0003-3355-2509) ${ }^{1}$
${ }^{1}$ Departamento de Psicologia, Escola Bahiana de Medicina e Saúde Pública. Av. Dom João VI 275, Brotas. 40290000 Salvador BA Brasil. laiskatharina@gmail.com ${ }^{2}$ Departamento de

Enfermagem, Escola Bahiana de Medicina e Saúde Pública. Salvador BA Brasil.

\begin{abstract}
The consolidation of practices aimed at enhancing resilience corroborates the current paradigm shift within the scope of adolescent health promotion, in which the individual's singularity is considered, as well as his/her relationship with the environment. The scope of the undertaking was to identify protective factors involved in the process of resilience among adolescents. This paper reviews indexed publications describing adolescent care models and interventions. The analysis of the texts consisted of: 1) comparing the similarities and differences between the concepts used; and 2) identifying, grouping and compiling the most cited protection factors. A total of 17 articles that strive to enhance resilience in six different practical contexts were analyzed. Based on the protection factors found, 17 actions were generated to guide the development of educational works in health. Protective factors related to "Problem solving", "Valorization of the individual" and "Ability in communication" were identified in several contexts of study on adolescence. These factors represent transversal aspects necessary to enhance resilience and should be stimulated together in order to foster the healthy development of adolescents. Key words Protective factors, Resilience, Adolescence
\end{abstract}

Resumo A consolidação de práticas voltadas para o fortalecimento da resiliência corrobora com a atual mudança de paradigmas no âmbito da promoção da saúde de adolescentes, em que se considera a singularidade do sujeito, assim como sua relação com o meio. Identificar fatores protetivos envolvidos no processo de resiliencia em adolescentes. Este trabalho revisa publicações indexadas que descrevem modelos e intervenções de atendimento ao adolescente. A análise dos textos consistiu em: 1) comparar as semelhanças e diferenças entre os conceitos utilizados; e 2) identificar, agrupar e compilar os fatores de proteção mais citados. Foram analisados 17 artigos que trabalham o fortalecimento da resiliência em seis contextos práticos distintos. A partir dos fatores de proteção encontrados foram geradas 17 ações norteadoras para desenvolvimento de trabalhos educativos em saúde. Fatores protetivos relacionado "Resolução de problemas", "Valorização do sujeito" e "Habilidade de comunicação" foram identificados em diversos contextos de estudo sobre adolescência. Esses fatores constituem aspectos transversais necessários ao fortalecimento da resiliência e devem ser estimulados conjuntamente a fim de favorecer o desenvolvimento saudável do adolescente.

Palavras-chave Fatores de proteção, Resiliência, Adolescência 


\section{Introdução}

A implementação de ações que visam o fortalecimento da resiliência entre adolescentes, principalmente dos que vivem em contextos adversos, surge como um avanço nas práticas protetoras da saúde ao provocar uma ênfase nas interações sociais e nos processos do desenvolvimento ${ }^{1}$. Tais ações resultam num olhar menos reducionista e individualista sobre $\mathrm{o}$ adolescente e mais direcionado para a saúde e não para a doença ${ }^{2}$.

No campo da saúde, a adolescência ainda é compreendida como uma etapa do desenvolvimento que se encontra em torno de diversos paradigmas e estereótipos naturalizantes. No início dos estudos sobre a adolescência havia a visão de que esta etapa era marcada por tormentos, conturbações, crises religiosas, contradições sucessivas entre outras características que a posicionam como algo abstrato e universal ${ }^{3}$. Atualmente, entretanto, discute-se sobre a adolescência ser uma construção social que repercute no desenvolvimento e na subjetividade do indivíduo. Reforçando essa ideia, o Conselho Federal de Psicologia, em 2002, apresentou na cartilha "Adolescência e Psicologia: concepções, práticas e reflexões críticas" a necessidade de superar a visão naturalizante e patologizante da adolescência, enfatizando a importância de enxergar a adolescência como concebida historicamente pelo ser humano, enquanto representação e fato social e psicológico ${ }^{4}$.

O momento da adolescência é subjetivo, portanto, a forma que o indivíduo vivencia esse período reflete em como vai se relacionar com as pessoas no seu ambiente. Neste contexto, fatores de risco ou fatores de proteção irão contribuir para o seu desenvolvimento e adoção (ou não) de um comportamento resiliente frente às adversidades encontradas na vida. Em meio a diversas conceituações sobre resiliência apresentadas na literatura, Assis et al. ${ }^{5}$ reúnem elementos que refletem melhor a amplitude desse processo. Para essa autora, resiliência é a capacidade de resistir às adversidades, é a força necessária para a saúde mental se estabelecer durante a vida, mesmo após a exposição a riscos; desse modo, significa a habilidade de se acomodar e se reequilibrar frente às adversidades.

A presença e o impacto, tanto dos fatores de risco como dos fatores de proteção, na vida dos indivíduos devem ser considerados como ações que visem à promoção da resiliência. O risco é um fator que ocasiona uma predisposição a um resultado negativo ${ }^{6}$ considerado unicamente em termos estatísticos e, sob essa perspectiva, os comportamentos de risco podem impactar a saúde ou o desempenho social do indivíduo ${ }^{7}$. O Ministério da Saúde ${ }^{8}$ elenca os fatores de risco para crianças e adolescentes em três grupos: ambiente social, domínio psicológico e domínio biológico.

Os fatores de proteção, por outro lado, são referidos como aqueles que diminuem a probabilidade de um resultado negativo ou indesejado ocorrer na presença de um fator de risco, podendo até reduzir a sua severidade ${ }^{6}$. Estes estão associados a características individuais ou ambientais que desempenham uma função de proteção, amparando o sujeito.

Antoni ${ }^{9}$ enfatiza a importância do desenvolvimento de pesquisas com crianças e adolescentes em situação de risco no intuito de identificar os aspectos saudáveis nesses grupos. Exemplos de situações que podem ser consideradas de risco, por afetarem o desenvolvimento do indivíduo, são: a ausência ou ineficácia do cuidado parental, os conflitos familiares (caso ocorram de maneira excessiva), a morte ou ausência abrupta de membro da família, pais ou cuidadores com algum transtorno mental, a vivência de violência doméstica, dentre outros ${ }^{10}$.

A literatura tem apontado para uma urgência em criar e incrementar políticas públicas que favoreçam ações para reparar e/ou minimizar os danos sofridos pelas adversidades na vida do adolescente $^{11}$. Com isso, diversos estudos têm sido realizados com o intuito de promover ou descrever estratégias que fomentem o desenvolvimento dos fatores de proteção, levando ao fortalecimento da resiliência ${ }^{12-15}$.

Este trabalho revisa publicações indexadas que descrevem modelos e intervenções sistematizadas de atendimento voltados à população adolescente, elencando os fatores de proteção apresentados em diversos contextos práticos, com objetivo de: (1) comparar semelhanças e diferenças entre conceitos utilizados pela pesquisa empírica sobre resiliência; (2) levantar os fatores protetivos mais citados pela pesquisa empírica sobre resiliência; e, (3) categorizar os fatores protetivos que apontam para ações educativas voltadas para a promoção da resiliência. Corresponde a um recorte de um projeto maior: "Educação em Saúde no Distrito Sanitário do Cabula/Beiru", desenvolvido pela Escola Bahiana de Medicina e Saúde Pública em parceria com a Universidade do Estado da Bahia (UNEB). 


\section{Método}

Trata-se de uma revisão de literatura que teve como pergunta norteadora: Quais os fatores protetivos envolvidos no processo de resiliência em adolescentes brasileiros?

Para identificação de artigos publicados em revistas científicas, foi consultada a base de dados Medical Literature Library of Medicine Online (Medline), Literatura Latino-Americana e do Caribe (Lilacs) e Biblioteca Virtual em Saúde dos Adolescente (AdolecBrasil) via Biblioteca Virtual em Saúde (BVS). Utilizaram-se os descritores: "resiliência" e "adolescência" ou "adolescente". Limitou-se a busca ao idioma português, além de selecionar as publicações que disponibilizavam textos completos e pesquisas empíricas, sem demarcação temporal.

Incluíram-se publicações abordando resiliência e respectivos fatores de proteção, dentro de um contexto específico: familiar, sexualidade, gênero, violência, situação de rua, desempenho escolar, medidas socioeducativas e vulnerabilidade social. Foram excluídos: (1) artigos que não condiziam com o tema da pesquisa; (2) artigos que não abordavam um desses contextos específicos; e, (3) estudos em duplicidade nas bases de dados ou de conteúdo (artigos transcritos na íntegra no corpo de dissertações publicadas nas mesmas bases de dados).

$\mathrm{Na}$ primeira etapa desse trabalho, o conceito de resiliência abordado nos artigos foi analisado e os conteúdos sobre esta temática foram sistematizados e categorizados a partir de aproximação, diferenças e complementações de ideias dos autores nos trabalhos. A partir desse resultado, foi possível obter a noção de como o conceito de resiliência está sendo trabalhado pelos profissionais de saúde nas intervenções práticas com adolescentes, no Brasil.

$\mathrm{Na}$ etapa seguinte, construiu-se uma ficha para análise de cada artigo, contendo: metodologia adotada, área dos profissionais envolvidos, concepção de resiliência presente no trabalho e os fatores de proteção fomentados nas intervenções. A partir da análise de cada trabalho incluído no estudo, os fatores de proteção apontados foram identificados e agrupados de acordo com o contexto específico (familiar, sexualidade, gênero, violência, situação de rua, desempenho escolar e medidas socioeducativas). Por fim, os fatores de proteção foram compilados e organizados de forma a elencar ações cujo propósito era de promover a resiliência em adolescentes, seja através da rede de apoio e/ou de intervenções interdisci- plinares. O fluxograma contendo as etapas dessa metodologia pode ser visto na Figura 1.

A linha teórica utilizada na concepção deste trabalho se baseia nas considerações de Rutter ${ }^{16}$, um clássico citado pela maioria dos estudos abordados neste trabalho, fato que demonstra a atualidade da sua contribuição. Este autor considera que os fatores protetivos podem ter quatro funções principais na vida de uma pessoa, quais sejam: reduzir o impacto dos riscos, o que altera a exposição da pessoa à situação adversa; reduzir as reações negativas em cadeia que seguem a exposição do indivíduo à situação de risco; estabelecer e manter a autoestima e autoeficácia, através da construção de relações de apego seguras e o cumprimento de tarefas com sucesso; e, criar oportunidades para reverter os efeitos do estresse. Desta forma, para análise de resultados, no presente estudo, foram considerados como fatores de proteção as ações e os aspectos que fomentassem esses quatro segmentos.

\section{Resultados e discussão}

A busca nas bases de dados resultou em 49 estudos, dos quais 32 foram excluídos: por serem duplicatas (dois), por possuírem seu conteúdo inserido em dissertações incluídas na pesquisa (dois), por abordarem o tema de forma insatisfatória, não contemplando conteúdos sobre resiliência e fatores de proteção (20), e por não apresentarem resiliência nos contextos específicos preestabelecidos (oito). Sobre o período de publicação, foram abarcados estudos de 1998 até 2014, sendo constatado que as publicações se duplicaram a partir de 2008 (Quadro 1).

Os 17 trabalhos analisados foram agrupados por áreas a partir do conteúdo abordado: "Gênero e família" (quatro), "Sexualidade" (três), "Violência em geral" (cinco) "Contexto sócio educativo" (um), "Desempenho escolar" (dois) e "Vulnerabilidade social" (dois). Esses dados podem ser verificados no Quadro 1.

Dos estudos encontrados, sete utilizaram métodos qualitativos de pesquisa, quatro foram estudos quantitativos, cinco foram revisões de literatura e um foi relato de experiência. A área de formação dos autores foi: Psicologia (11), Enfermagem (dois) e Medicina (um), sendo que três trabalhos foram desenvolvidos por equipe interdisciplinar. Esse fato sugere que, embora haja consenso acerca da importância da abordagem interdisciplinar na promoção da saúde do adolescente $^{17}$, o tema resiliência ainda é pouco 


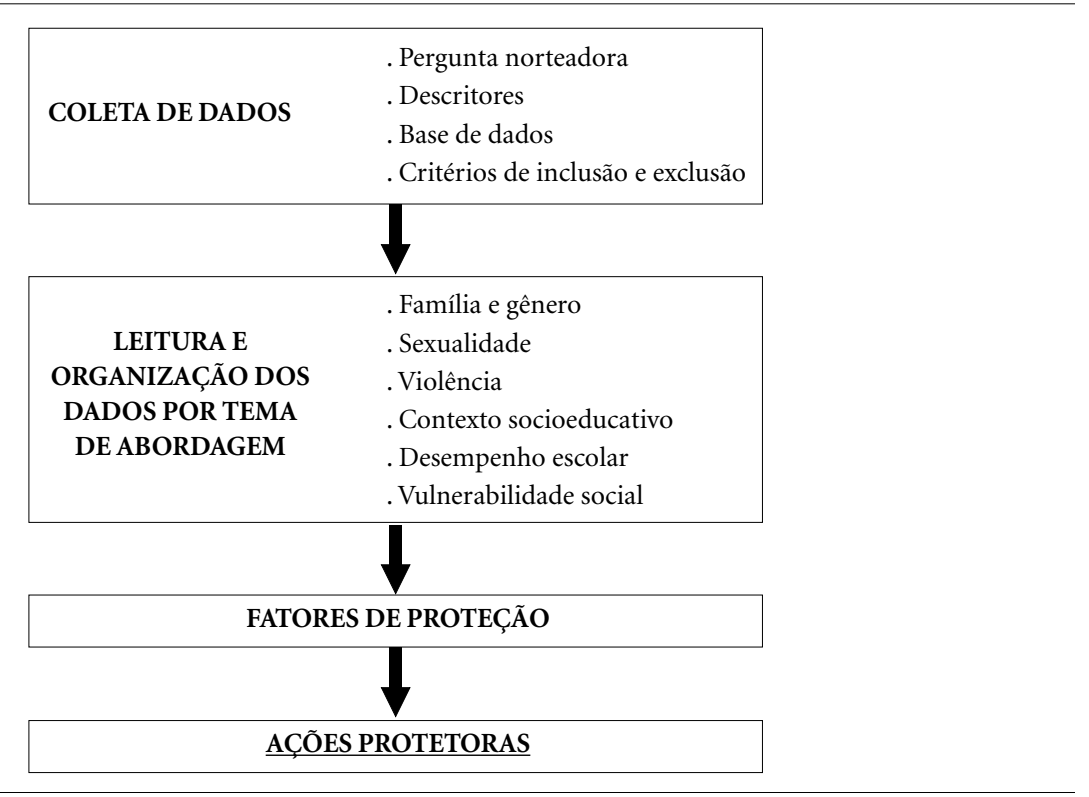

Figura 1. Fluxograma contendo as etapas metodológicas desta pesquisa.

Quadro 1. Levantamento de publicações abordando resiliência dentro de contextos específicos.

\begin{tabular}{|c|c|c|}
\hline Contexto & Artigo & Autores \\
\hline \multirow{4}{*}{$\begin{array}{l}\text { Família e } \\
\text { Gênero }\end{array}$} & Aperfeiçoar a resiliência de adolescentes e suas famílias & Barankin $^{12}$ \\
\hline & $\begin{array}{l}\text { Considerações sobre a resiliência de adolescentes filhos de alcoolistas no } \\
\text { contexto familiar }\end{array}$ & Trindade e Costa ${ }^{27}$ \\
\hline & $\begin{array}{l}\text { Resiliência na adolescência: uma reflexão sobre o potencial de superação } \\
\text { de problemas em meninos e meninas }\end{array}$ & Rozemberg $^{11}$ \\
\hline & $\begin{array}{l}\text { O impacto do ambiente familiar nos primeiros anos de vida: um estudo } \\
\text { com adolescentes de uma invasão de Salvador, Bahia }\end{array}$ & Bastos et al. ${ }^{18}$ \\
\hline \multirow[t]{3}{*}{ Sexualidade } & $\begin{array}{l}\text { Programa de habilidades interpessoais e direitos sexuais e reprodutivos } \\
\text { para adolescentes: um relato de experiência }\end{array}$ & Murta et al. ${ }^{19}$ \\
\hline & Gestação na adolescência: a construção do processo saúde-resiliência & Ribeiro e Gualda ${ }^{28}$ \\
\hline & $\begin{array}{l}\text { Percursos da gravidez na adolescência: estudo longitudinal após uma } \\
\text { década da gestação }\end{array}$ & $\begin{array}{l}\text { Oliveira- } \\
\text { Monteiro }^{29}\end{array}$ \\
\hline \multirow[t]{5}{*}{ Violência } & Fatores de risco e de proteção em adolescentes vítimas de abuso sexual & Borges e Zingler ${ }^{13}$ \\
\hline & Abuso sexual e resiliência: enfrentando as adversidades & Martins $^{24}$ \\
\hline & $\begin{array}{l}\text { Apego em adolescentes institucionalizadas: processos de resiliência na } \\
\text { formação de novos vínculos afetivos }\end{array}$ & $\begin{array}{l}\text { Dell'Aglio e } \\
\text { Dalbem }^{30}\end{array}$ \\
\hline & $\begin{array}{l}\text { Prostituição de adolescentes: uma imagem construída na adversidade da } \\
\text { sociedade }\end{array}$ & Botelho $^{25}$ \\
\hline & $\begin{array}{l}\text { Violência e resiliência: a criança resiliente na adversidade [temas em } \\
\text { debate] }\end{array}$ & Grunspun $^{22}$ \\
\hline $\begin{array}{l}\text { Contexto } \\
\text { socioeducativo }\end{array}$ & $\begin{array}{l}\text { Fatores protetivos a adolescentes em conflito com a lei no contexto } \\
\text { socioeducativo }\end{array}$ & Costa e Assis ${ }^{14}$ \\
\hline \multirow[t]{2}{*}{$\begin{array}{l}\text { Desempenho } \\
\text { Escolar }\end{array}$} & $\begin{array}{l}\text { Atribuições de causalidade para o desempenho escolar e resiliência em } \\
\text { estudantes }\end{array}$ & $\begin{array}{l}\text { Garcia e } \\
\text { Boruchovitch }^{23}\end{array}$ \\
\hline & $\begin{array}{l}\text { Eventos estressores, estratégias de coping e desempenho escolar em } \\
\text { adolescentes }\end{array}$ & Busnello et al. $^{32}$ \\
\hline \multirow{2}{*}{$\begin{array}{l}\text { Vulnerabilidade } \\
\text { Social }\end{array}$} & Resiliência na rua: um estudo de caso & Paludo e Koller $^{20}$ \\
\hline & $\begin{array}{l}\text { Adolescentes e jovens em situação de risco psicossocial: redes de apoio } \\
\text { social e fatores pessoais de proteção }\end{array}$ & Amparo et al. ${ }^{15}$ \\
\hline
\end{tabular}


explorado nessa perspectiva. O fortalecimento de equipes interdisciplinares dentro das redes de apoio que cercam o adolescente é de fundamental importância para o avanço de práticas efetivas nessa área de tamanha complexidade.

O bom entendimento do conceito de resiliência é vital para o estabelecimento de práticas consistentes e efetivas que visem fomentar fatores protetivos à saúde de adolescentes. Dos 17 artigos desta revisão, cinco versaram sobre fatores de proteção sem apresentar, explicitamente, o conceito de resiliência adotado ${ }^{11,15,18-20}$. De acordo com a literatura, o conceito de resiliência é, muitas vezes, confundido com o de fatores de proteção ${ }^{21} \mathrm{e}$, desta forma, nesse estudo buscou-se compreender as concepções adotadas por profissionais que já atuam com intervenções nesta abordagem. Semelhanças, aproximações e diferenças nos conceitos adotados nos artigos foram reunidas de forma sintética e sistemática a fim de possibilitar aos profissionais um aporte teórico com um conteúdo diretivo e acessível. As características do conceito de resiliência abordados nos artigos incluídos nesta pesquisa foram sistematizadas em quatro categorias, sendo que resiliência pode ser considerada como:

- Habilidade, potencial ou capacidade do ser humano, família ou grupo social em superar as situações estressoras e adversas, criando estratégias psicológicas de enfrentamento para passar por essas situações sem se submeter às mesmas, vivenciando um desenvolvimento saudável após a exposição a riscos, recuperando-se e mantendo um comportamento adaptado após um dano, dificuldade ou mudança. Pode, também, prevenir, minimizar e superar os efeitos nocivos das adversidades, reconstruir e continuar sua trajetória de vida de forma mais positiva e favorável. $\mathrm{O}$ termo "superação de adversidades", comumente associado a resiliência, não significa que a pessoa escape completamente ilesa de situações consideradas muito estressantes. A possibilidade de superação por meio da ressignificação do problema é ressaltada e, desta forma, considera-se que o indivíduo seja fortalecido ou transformado pelas adversidades ${ }^{11,13-15,22,23}$.

- Um fenômeno que ocorre na interação entre o risco/vulnerabilidade e a proteção no modo como estes se encontram presentes nos diversos contextos do desenvolvimento humano. Os fatores protetivos são compreendidos como elementos que modificam ou alteram a resposta de uma pessoa ao perigo ambiental; não eliminam os riscos ou tornam o sujeito "invulnerável”, mas encorajam o indivíduo a lidar, efetivamente, com a situação de risco e sair fortalecido da mesma, permitindo ter um desenvolvimento sadio mesmo que este esteja vivendo em um ambiente desfavorável. Ou seja, a resiliência refere-se a processos que operam na presença de risco para produzir consequências boas ou melhores do que aquelas obtidas na ausência do risco ${ }^{12-15,24,25}$.

- Determinada pela tríade atributos individuais, família e fatores ambientais/sociais, tríade que se assemelha ao modelo ecológico-transacional de Bronfenbrenner ${ }^{26}$, o qual compreende os níveis que formam o marco ecológico: individual, familiar, comunitário e cultural. Sob essa perspectiva, a resiliência é construída de forma sócio histórica, atribuída de valores culturais e sociais específicos do ambiente e correspondendo à combinação dos atributos que a pessoa possui, bem como do meio social e cultural em que está inserida ${ }^{11,24,25,27}$.

- Um processo dinâmico, uma vez que a resiliência não deve ser vista como um conceito estagnado e sob um enfoque individualista ou como um evento isolado, não se constituindo de uma capacidade humana naturalizada e estática. Corresponde a um processo que deve sempre ser entendido e relativizado como simultaneamente social e intrapsíquico - não é um traço imutável, estando em constante mudança, é dinâmico. Assim sendo, não existe uma pessoa que é resiliente, mas sim a que está resiliente, podendo ser continuamente incrementada ao longo do desenvolvimento sendo aprendida e estimulada. Está presente no indivíduo de todas as idades, nas comunidades, na sociedade e nas organizações, podendo também ser desenvolvida no ser humano, especialmente nas crianças, já que está intimamente relacionada ao desenvolvimento e ao crescimento humanos ${ }^{11,13,14,22-25,27-30}$.

- Uma condição que pode ser desenvolvida e estimulada, pois os autores pioneiros enfatizavam que estes atributos individuais poderiam ser estimulados em pessoas que não conseguem desenvolvê-los espontaneamente. Algumas características da pessoa resiliente são a noção de controle, a capacidade de relacionamento e a relatividade emocional. Existe um modelo capaz de caracterizar o perfil da resiliência através das expressões "eu tenho, eu sou, eu estou, eu posso". Não está condicionada ao nível socioeconômico e constitui uma dimensão importante para a saúde mental e para a qualidade de vida. A resiliência é mais do que apenas sobreviver, uma vez que os sobreviventes nem sempre são, necessariamente, dotados de "boa resiliência"; alguns ficam presos alimentando seus sofrimentos, sendo, desta for- 
ma, necessário dar ênfase ao aspecto positivo do enfrentamento e da adaptação $0^{23-25,27,29}$.

A fim de contribuir com o desenvolvimento de novos trabalhos educativos em saúde no âmbito do fortalecimento da resiliência de adolescentes e jovens, ações norteadoras foram levantadas a partir dos fatores de proteção enfatizados nos artigos desta revisão. As ações foram delineadas e agrupadas em três eixos temáticos: "Resolução de problemas", "Valorização do sujeito" e "Habilidade de comunicação" (Quadros 2 a 4).

Encorajar o adolescente a desenvolver capacidades e habilidades para resolução de problemas constitui um fator de proteção que requer estímulo a partir de diferentes tipos de ações. Esta categoria reúne aspectos como o insight, compreensão do conceito de resiliência, adaptação a novas realidades, enfretamento de adversidades (estratégias de coping), autonomia, aproximação do adolescente ao serviço de saúde e realização de sonhos, desejos e projetos.

$\mathrm{O}$ insight possibilita que o sujeito identifique o padrão de comportamento a que está submetido, ao mesmo tempo em que permite a compreensão do motivo pelo qual este comportamento acontece $^{12,31}$. Desta forma, favorecer o insight, isto é, a percepção do problema como um problema, é fundamental para a elaboração do sofrimento e da superação da adversidade.

Em um estudo feito por Antoni ${ }^{9}$ com meninas que sofreram maus tratos em contexto familiar, a presença do insight possibilitou a percepção acerca da repetição da violência, favorecendo maiores condições para o rompimento desse ciclo e evitando maus tratos das próximas gerações. A autora salienta que refletir sobre a sua realida-

Quadro 2. Propostas de ações norteadoras para favorecer o desenvolvimento do fator de proteção resolução de problemas.

\begin{tabular}{|c|}
\hline Resolução de Problema \\
\hline $\begin{array}{l}\text { 1. Propiciar uma forma de aprender e apreender a identificar ou reconhecer situações geradoras de opressão e } \\
\text { possíveis comportamentos relativos a elas: insight }{ }^{12} \text {; } \\
\text { 2. Informar sobre o que é resiliência e como ela pode ser promovida }{ }^{19} \text {; } \\
\text { 3. Encorajar o enfrentamento às adversidades, favorecendo o desenvolvimento de estratégias e competências } \\
\text { para lidar com o estresse e resolver problemas, favorecendo o desenvolvimento de estratégias de coping }{ }^{11,32} \text {; } \\
\text { 4. Estimular a autonomia através de atividades de compartilhamento, reciprocidade e responsabilidade social } \\
\text { através de tomada de decisão e de comprometimento com o bem-estar individual e coletivo }{ }^{12,23,28,29} \text {; } \\
\text { 5. Despertar para atribuições de causalidades compatíveis com a motivação para o aprendizado e para a } \\
\text { superação de dificuldades }{ }^{14} \text {; } \\
\text { 6. Incentivar postura otimista através da contraposição do pensamento negativo com uma forma mais positiva } \\
\text { e otimista de avaliar a situação }{ }^{12,28} \text {; } \\
\text { 7. Estimular e favorecer a aproximação do adolescente ao serviço de saúde, a fim de fornecer recursos teóricos } \\
\text { e emocionais para adaptação às novas realidades de vida - amadurecimento e responsabilidade, visando a } \\
\text { compreensão da repercussão dos seus atos e consequências }{ }^{11,13,29} \text {; } \\
\text { 8. Encorajar a refletir sobre seus sonhos, desejos e projetos, discutindo recursos, estratégias e passos necessários } \\
\text { e metas positivas (autodisciplina) se concretizem }{ }^{13,19,22} \text {. }\end{array}$ \\
\hline
\end{tabular}

Quadro 3. Propostas de ações norteadoras para favorecer o desenvolvimento do fator de proteção valorização do sujeito.

\begin{tabular}{|l|}
\multicolumn{1}{|c|}{ Valorização do Sujeito } \\
\hline 1. Promover o fortalecimento da autoestima ${ }^{12,13,20,22,27,28}$; \\
2. Incentivar a desenvolver um sentido de competência baseado em seus talentos e habilidades, \\
proporcionando experiências variadas e estimulantes apropriadas para a idade, tais como: feira de ciência, \\
concurso de soletração, atividades esportivas ${ }^{12}$; \\
3. Estimular a inserção em algum de sistema de crenças espirituais ${ }^{12,13,22,29,32}$; \\
4. Favorecer o pensamento crítico sobre questões de gênero e os direitos sexuais e reprodutivos na \\
adolescência $^{11}$; \\
5. Estimular ações de autocuidado, tais como: alimentação, prevenção de doenças, atividades físicas e de lazer \\
saudáveis
\end{tabular}


Quadro 4. Propostas de ações norteadoras para favorecer o desenvolvimento do fator de proteção habilidade de comunicação.

Habilidade de Comunicação

1. Oportunizar espaços de desenvolvimento da habilidade de expressar sentimentos de forma assertiva ${ }^{12,19,22,27}$.

2. Estimular a utilização de técnicas de relaxamento e controle da raiva e do temperamento ${ }^{12}$;

3. Estimular o relacionamento íntimo e protetor com um adulto (inclusos adultos do círculo social/familiar e rede formal de apoio - educação e saúde) $)^{11-13,19,22,24,27}$;

4. Auxiliar no estabelecimento de relações sócio afetivas dotadas de mais qualidade ${ }^{11-13,18,20,22,24,27-29,32}$ :

. (com pai e mãe) conscientizando da importância e promovendo, quando possível, a compreensão e valorização da figura materna e paterna;

. (com adulto responsável) estimular e facilitar a procura e abertura para construção e manutenção de relacionamentos com amigos ou pessoas significativas que possam assumir papel de referência e de segurança nos momentos de adversidade ao longo da vida; e,

. (com os pares) promover o estabelecimento de relações interpares através do respeito ao outro e da

valorização de si.

de, de maneira consciente, poderá auxiliar estas meninas a buscarem relações com maior reciprocidade, afetividade e estabilidade. Os momentos da pesquisa que provocaram insights nas participantes auxiliaram na promoção da resiliência individual de algumas delas.

Nas ações educativas descritas por Murta et al. ${ }^{19}$, o tema "Identidade e Resiliência" foi trabalhado em uma das primeiras sessões, no momento em que os adolescentes foram informados sobre o conceito de resiliência e como esta pode ser fortalecida. Esta intervenção se baseou na exposição dialogada, na psicoeducação e na atividade para representação da resiliência através de fatores de proteção, pessoas e experiências de superação de problemas. Apresentar ao adolescente o comportamento resiliente como uma forma de se reagir a um problema possibilita a identificação e a busca pelos aspectos positivos de proteção, tirando-se a ênfase dos fatores de risco.

Adotar estratégias de coping foi uma das formas de enfrentamento de problemas encontrada nessa revisão ${ }^{11,32}$, e depende de fatores inerentes ao indivíduo e ao ambiente. É necessário aprender a usar recursos cognitivos e comportamentais a fim de perceber o problema, avaliar as possíveis vias de resolução e mobilizar-se para, de forma mais apropriada, administrar a situação estressora $^{33}$. Diniz e Zanini ${ }^{34}$ demonstraram a importância da avaliação que o adolescente faz sobre os eventos adversos, evidenciando a sua influência na escolha das estratégias de enfrentamento.

O desenvolvimento da autonomia é outro aspecto importante para a capacidade de resolução de problemas. A realidade social, política, econômica e cultural em que o adolescente está inserido interfere na maneira como as famílias vão incentivar ou dificultar o desenvolvimento da autonomia ${ }^{35}$. Tanto o contexto social quanto o familiar, se desfavoráveis, podem ser apontados pelos adolescentes como causas de incapacidade e de insucessos para resolver os problemas da vida cotidiana. A compreensão das relações entre a atribuição de causalidade e o comportamento futuro do indivíduo pode favorecer programas de intervenção que alterem as atribuições causais que se mostram incompatíveis com a motivação e com o otimismo necessários a uma postura ativa e autônoma diante dos problemas ${ }^{12,23,28,29,32}$.O estímulo da autonomia pode e deve ser inserido em programas de saúde na Atenção Básica ${ }^{36}$.

O serviço de saúde deveria ser compreendido pelos adolescentes como um relevante suporte que oferece recursos teóricos e emocionais para a sua adaptação às novas realidades de vida, além dos recursos puramente clínicos ${ }^{11,13,29,37,38}$. A dificuldade em acolher os adolescentes por parte dos profissionais, entretanto, tem despertado iniciativas de profissionais e de gestores que atuam nessa área.

$\mathrm{Na}$ Bahia, Cícero et al. ${ }^{39}$, no grupo de educação em saúde "Adolescer com Arte", desenvolveram ações com a finalidade de promover o protagonismo juvenil através de atividades voltadas para artes com ênfase em música e teatro. Este projeto foi proposto por um agente comunitário de saúde como resposta à baixa aderência dos adolescentes à Unidade de Saúde (US). Uma das dificuldades iniciais apontadas pelos autores foi o fato de que os próprios profissionais de saúde não conseguiam aceitar a realização das atividades do grupo dentro da US. Assim, após quatro meses de projeto, foi observado pelos profissionais da US o aumento do número de jovens que procuraram o serviço. 
Conseguir demarcar estratégias para resolução de problemas auxilia o adolescente a pensar em projetos de vida e vice-versa, pois quando o jovem pensa sobre o seu futuro, o desejo da realização tende a alimentar a motivação para ultrapassar obstáculos ${ }^{13,19,22}$. No âmbito escolar, Nascimento ${ }^{40}$ ressalta que planejar o futuro auxilia no desenvolvimento da consciência crítica e reflexiva, bem como do aprendizado do saber fazer por parte dos adolescentes. Murta et al. ${ }^{19}$, em uma das sessões do programa de habilidades interpessoais e direitos sexuais e reprodutivos, incentivaram a discussão sobre o enfrentamento de adversidades, fazendo com que os adolescentes refletissem sobre o seu futuro, escrevendo os recursos necessários para isso e conversando sobre $\mathrm{o}$ assunto com um amigo.

Alguns problemas enfrentados por crianças e adolescentes obstaculizam o desenvolvimento do potencial de resiliência por amortizarem a confiança e a estima por $\mathrm{si}^{5}$. No Quadro 3 foram agrupados fatores protetivos voltados para a valorização do sujeito adolescente e para a promoção do cuidado de si, englobando o autocuidado, o investimento em programas de lazer, as reflexões sobre a espiritualidade, o desenvolvimento e o reconhecimento de habilidades e competências em potenciais, e avaliação das situações da vida de forma mais positiva.

Um espaço propício para que o jovem seja capaz de se apropriar dos seus recursos internos e subjetividade, quer seja no ambiente familiar, na escola, ou em outro lugar, é essencial para o desenvolvimento da autoestima, assim como para o exercício da autonomia.

A autoestima é um aspecto que afeta a participação e a integração positiva de um adolescente com outros (no grupo) em um projeto ${ }^{12,13,20,22,27,28}$. Adolescentes com menor autoestima apresentam maior dificuldade para integração grupal, podendo desenvolver mecanismos que, provavelmente, distorcem a comunicação de seus sentimentos e pensamentos que dificultam, ainda mais, essa integração $0^{41}$. Um dos fatores individuais para fomentar a resiliência seria incentivar o desenvolvimento significativo de competências baseado em talentos e habilidades do jovem ${ }^{12}$. Compreendese que, desse modo, $\mathrm{o}$ adolescente passaria a perceber e a utilizar essas qualidades sendo mais um passo para a valorização do sujeito.

A manutenção de uma crença religiosa constitui outro aspecto que influencia a valorização de $\operatorname{si}^{12,13,22,29,32}$. A religiosidade foi considerada como um fator protetivo de adolescentes mães durante o processo da construção da maternida- $\mathrm{de}^{29}$. Outros estudos apontaram que a aderência a um movimento religioso incrementa a rede de apoio social e afetiva, impactando positivamente na relação entre pais e filhos adolescentes ${ }^{42}$, possibilitando melhor controle dos impulsos e maior bem-estar, o que contribui, ainda, para a melhor estabilidade na saúde mental e no melhor enfretamento em situações estressantes ${ }^{43}$.

Em relação às questões de gênero, Rozemberg $^{11}$ realizou pesquisa com 889 adolescentes a fim de identificar as diferenças do potencial de resiliência de adolescentes do sexo feminino e masculino. Essa autora não encontrou diferenças significativas, exceto a necessidade de priorizar um espaço individualizado para as meninas. Segundo a autora, a conquista de um espaço próprio que proporcione tranquilidade às meninas parece ser importante para o desenvolvimento da sua resiliência. Em relação aos meninos, o estudo revelou que o trabalho fora de casa pode constituir um fator de proteção, apesar de ser necessário considerar o efeito negativo sobre o desempenho escolar. De acordo com esses autores, o trabalho fora de casa pode "ser um fator positivo para as relações sociais e para um melhor desempenho em atividades, além de representar oportunidade de contato com mais pessoas fora do contexto familiar".

Murta et al. ${ }^{19}$, em um programa de promoção de habilidades interpessoais e direitos sexuais e reprodutivos para adolescentes, abordou o desenvolvimento de atitudes mais flexíveis acerca dos papéis de gênero, possibilitando maneiras de se relacionar mais respeitosas e adequadas em relação ao próprio direito, ao direito do outro e à diversidade humana.

Práticas relacionadas ao autocuidado têm sido o alvo de grande parte das intervenções educativas em saúde do adolescente, frequentemente focadas na prevenção de doenças e riscos inerentes a essa população. No contexto da valorização do sujeito, o incentivo para a realização de atividades físicas, atividades de lazer coletivas e animadas e a escolha de práticas alimentares saudáveis correspondem a uma forma de prevenir doenças e de promover saúde mental ${ }^{12,29}$.

A comunicação interpessoal constitui-se como um alicerce para a formação de redes de troca social, mantendo e alterando a realidade social e cultural do adolescente. Repercute, assim, no bem-estar, na autoestima, na qualidade das relações familiares e interpares e na integração social de maneira geral ${ }^{44}$. A habilidade de comunicação corresponde a um recurso individual do comportamento resiliente que favorece a resolu- 
ção de problemas, quer seja diretamente ou por facilitar o acesso à rede de apoio. Além disso, é através da relação interpessoal, mediada pela comunicação, que o indivíduo se percebe como um sujeito com seus valores e limitações. Estudos encontrados nesta revisão abordaram a importância da habilidade de comunicação no contexto da resiliência. Adolescentes descritos como resilientes conseguiram confiar em alguém e falar sobre seus sentimentos, assim como adquiriram segurança e determinação para dizer "não", quando necessário ${ }^{12,19,27,22}$.

Dois programas educativos, já citados neste trabalho ${ }^{19,36}$, abordaram o tema comunicação enfatizando aspectos relacionados à expressão de sentimentos. No primeiro ${ }^{19}$, salientou-se o uso de comunicação assertiva para a redução de riscos diversos como: depressão, uso de drogas, gravidez precoce e suicídio; a necessidade de identificação e manejo de emoções desagradáveis, como raiva, ansiedade e tristeza; o aprendizado de formas de expressar seus sentimentos "sem machucar" o outro e de solicitar ajuda a pessoas de confiança. No segundo ${ }^{36}$, foram discutidas situações geradoras de sentimentos negativos como a raiva, o medo, a ansiedade e a tristeza; nesse programa, os jovens foram motivados ao uso de estratégias saudáveis para expressar estes sentimentos. É legítima a necessidade de espaços e de programas como esses, nos quais se torna possível ao adolescente não apenas expressar seu sentimento, mas aprender formas de fazê-lo positivamente ${ }^{12}$.

Um dos ganhos do desenvolvimento de habilidade de expressar sentimentos está em estabelecer relações sócio afetivas dotadas de mais qualidade, além da eficácia para conseguir solicitar ajuda a um adulto em situação de crise 11-13,18,20,22,24,27-29,32 $^{\text {. }}$ Em casos de abuso sexual, o suporte familiar foi fundamental para a superação de eventos estressores, promovendo um ambiente propício para a expressão da situação, além de apoiar o jovem para que o mesmo não se sinta culpado pelo evento de violência ${ }^{13}$. As figuras materna e paterna têm destaque neste lugar de cuidado, favorecendo a manutenção de um equilíbrio e/ou a redução de danos psicológicos nos seus filhos ${ }^{27}$. O espaço familiar deveria ser aquele no qual os jovens se identificam, se defendem e encontram apoio para superar as situações adversas.

\section{Considerações finais}

Por décadas, o senso comum vem reduzindo e estigmatizando a adolescência como uma fase de riscos e vulnerabilidades, na qual se enfatizam dados referentes às infrações, uso de substâncias psicoativas, doenças sexualmente transmissíveis e gravidez não planejada. $\mathrm{Na}$ contramão desse rótulo patologizante, entretanto, tem crescido o número de fóruns sociais e ações voltadas para a promoção da saúde do adolescente com vistas à valorização da subjetividade de cada jovem, assim como, à garantia dos seus direitos básicos e à superação de adversidades.

Nesse cenário, a proposta deste estudo foi identificar em publicações científicas brasileiras os fatores protetivos favoráveis ao fortalecimento da resiliência de adolescentes considerados pelos respectivos autores em contextos específicos, tais como família e gênero, sexualidade, violência, contexto sócio educativo, desempenho escolar e vulnerabilidade social.

A partir de semelhanças, aproximações e diferenças entre os conceitos de resiliência adotados nos artigos incluídos na revisão, foi possível concluir que esta corresponde à habilidade para criar e utilizar estratégias que possibilitem a ressignificação das situações adversas encontradas na vida, favorecendo a superação e o fortalecimento por meio destas. Dessa forma, a resiliência se dá a partir da interação entre a vulnerabilidade e a proteção, determinada por atributos individuais, familiares e sociais, num processo dinâmico, que deve ser desenvolvido e estimulado. Considerar resiliência como sinônimo de resignação, resistência ou adaptação é simplista e comprometedor.

Fatores protetivos relacionados à "Resolução de problemas", "Valorização do sujeito" e "Habilidade de comunicação" foram identificados nos diversos contextos estudados sobre adolescência, convertidos em ações norteadoras e sugeridos para o planejamento e desenvolvimento de novas abordagens promotoras da saúde com foco no fortalecimento da resiliência. Esses fatores constituem aspectos transversais necessários ao fortalecimento da resiliência e devem ser estimulados conjuntamente a fim de favorecer o desenvolvimento saudável do adolescente dentro de qualquer setor da rede de apoio a este relacionado, quer seja família, professores e funcionários das escolas, rede de saúde, instituições comunitárias, organizações não governamentais, dentre outros. 


\section{Colaboradores}

LKP Santos e CC Santana trabalharam em todas as etapas da elaboração deste manuscrito (concepção, metodologia, pesquisa, tabulação e análise dos dados e redação final). MVO Souza colaborou no desenvolvimento metodológico e na redação final.

\section{Referências}

1. Yunes MAM. Psicologia positiva e resiliência: o foco no indivíduo e na família. Psicologia em Estudo 2003; 28(n. esp.):75-84.

2. Infante F. A resiliência como processo: uma revisão da literatura recente. In: Melillo A, Ojeda ENS, organizadores. Resiliência: descobrindo as próprias fortalezas. São Paulo: Artmed; 2005. p. 22-38.

3. Aberastury A, Knobel M. Adolescência normal. Porto Alegre: Artmed; 1989.

4. Ozella S. Adolescência e Psicologia: concepções, práticas e reflexões críticas. Brasília: MS; 2002.

5. Assis SG, Avanci JQ, Pesce RP, Njaine K. Resiliência na adolescência: refletindo com educadores sobre superação de dificuldades. Rio de Janeiro: Fiocruz; 2008.

6. Cowan PA, Cowan CP, Schulz MS. Thinking about risk and resilience in families. In: Hetherington EM, Blechman EA, organizadores. Stress, coping and resiliency in children and families. Mahwah: Lawrence Erlbaum; 1996. p. 1-38.

7. Reppold CT, Pacheco J, Bardagi M, Hutz CS. Prevenção de problemas de comportamento e desenvolvimento de competências psicossociais em crianças e adolescentes: uma análise das práticas educativas e dos estilos parentais. In: Hutz CS, organizador. Situações de risco e vulnerabilidade na infância e adolescência: aspectos teóricos e estratégias de intervenção. São Paulo: Casa do Psicólogo; 2002. p. 7-51.

8. Brasil. Ministério da Saúde (MS). Secretaria de Atenção à Saúde. Área de Saúde do Adolescente e do Jovem. Marco legal: saúde, um direito de adolescentes. Brasília: MS; 2005.

9. Antoni C. Vulnerabilidade e resiliência familiar na visão de adolescentes maltratadas [dissertação]. Porto Alegre: Universidade Federal do Rio Grande do Sul; 2000.

10. Brasil. Ministério da Saúde (MS). Secretaria de Atenção à Saúde. Departamento de Atenção Básica. Saúde mental. Brasília: MS; 2013.

11. Rozemberg LB. Resiliência na adolescência: uma reflexão sobre o potencial de superação de problemas em meninos e meninas [tese]. Rio de Janeiro: Fiocruz, ENSP 2014.

12. Barankin T. Aperfeiçoar a resiliência de adolescentes e suas famílias. Adolesc Saúde 2013; 10(2):17-22.

13. Borges JL, Zingler VT. Fatores de risco e de proteção em adolescentes vítimas de abuso sexual. Psicol Estud 2013; 18(3):453-463.

14. Costa CRBSF, Assis SG. Fatores protetivos a adolescentes em conflito com a lei no contexto socioeducativo. Psicol Soc 2006; 18(3):74-81.

15. Amparo DM, Galvão ACT, Alves PB, Brasil KT, Koller $\mathrm{SH}$. Adolescentes e jovens em situação de risco psicossocial: redes de apoio social e fatores pessoais de proteção. Estud Psicol 2008; 13(2):165-174.

16. Rutter M. Psychosocial resilience and protective mechanisms. Am J Orthopsychiatry 1987; 57(3):316-331. 
17. Brasil KCT, Amparo DM, Gusmão MM, Oliveira RM, Medeiros MO, Novaes C, Belfort L. O trabalho interdisciplinar no contexto da exclusão. Psicol Cienc Prof 2003; 23(3):90-97.

18. Bastos ACS, Urpia ACM, Pinho L, Almeida Filho NM. O impacto do ambiente familiar nos primeiros anos de vida: um estudo com adolescentes de uma invasão de Salvador, Bahia. Estudos de Psicologia 1999; 4(2):239-271.

19. Murta SG, Ribeiro DC, Rosa IO, Menezes JCL, Rieiro MRS, Borges OS, Paulo SG, Oliveira V, Miranda VH, Prette AD, Prette ZAPD. Programa de habilidades interpessoais e direitos sexuais e reprodutivos para adolescentes: um relato de experiência. Psico USF 2012; 17(1):21-32.

20. Paludo SS, Koller SH. Resiliência na rua: um estudo de caso. Psic Teor Pesq 2005; 21(2):187-195.

21. Sapienza G, Pedromônico MRM. Risco, proteção e resiliência no desenvolvimento da criança e do adolescente. Psicol Estud 2005; 10(2):209-216.

22. Grunspun H. Violência e resiliência: a criança resiliente na adversidade. Bioética 2002; 10(1):163-171.

23. Garcia NR, Boruchovitch E. Atribuições de causalidade para o desempenho escolar e resiliência em estudantes. Psico USF 2014; 19(2):277-286.

24. Martins RC. Abuso sexual e resiliência: enfrentando as adversidades. Rev Mal-Estar Subj 2011; 11(2):727-750.

25. Botelho SMN. Prostituição de Adolescentes: uma imagem construída na adversidade da sociedade [tese]. São Paulo: Universidade de São Paulo; 2003.

26. Bronfenbrenner U. A ecologia do desenvolvimento humano: experimentos naturais e planejados. Porto Alegre: Artes Médicas; 1996.

27. Trindade EMV, Costa LF. Considerações sobre a resiliência de adolescentes filhos de alcoolistas no contexto famíliar. Comun Cien Saúde 2012; 23(2):179-188.

28. Ribeiro PM, Gualda DMR. Gestação na adolescência: a construção do processo Saúde-Resiliência. Esc Anna Nery 2011; 15(2):361-371.

29. Oliveira-Monteiro NR. Percursos da gravidez na adolescência: estudo longitudinal após uma década da gestação. Psicol Reflex Crit 2010; 23(2):278-288.

30. Dell'Aglio DD, Dalbem JX. Apego em adolescentes institucionalizadas: processos de resiliência na formação de novos vínculos afetivos. Psico 2008; 39(1):3340.

31. Abel MC. O insight na psicanálise. Psicol Cien Prof 2003; 23(4):22-31.

32. Busnello FB. Eventos estressores, estratégias de coping e desempenho escolar em adolescentes [dissertação]. Porto Alegre: PUC Rio Grande do Sul; 2009.

33. Raimundo RCP, Pinto MAPM. Stress e estratégias de coping em crianças e adolescentes em contexto escolar. Aletheia [online] 2006; 24:9-19.

34. Diniz SS, Zanini DS. Relação entre fatores de personalidade e estratégias de coping em adolescentes. Psico USF 2010; 15(1):71-80.
35. Reichert CB, Wagner A. Autonomia na adolescência e sua relação com os estilos parentais. Psico 2008; 38(3):292-299.

36. Silva MP, Murta SG. Treinamento de habilidades sociais para adolescentes: uma experiência no programa de atenção integral à família (PAIF). Psicol Reflex Crit 2009; 22(1):136-143.

37. Claro LBL, March C, Mascarenhas MTM, Castro IAB, Rosa MLG. Adolescentes e suas relações com serviços de saúde: estudo transversal em escolares de Niterói, Rio de Janeiro, Brasil. Cad Saúde Pública 2006; 22(8):1565-1574.

38. Morais NA, Morais CA, Reis S, Koller SH. Promoção de saúde e adolescência: um exemplo de intervenção com adolescentes em situação de rua. Psicol Soc 2010; 22(3):507-518

39. Cícero LB, Santana CC, Carmo GCS, Carvalho RC. Adolescer com arte: um exemplo de protagonismo juvenil. Adolesc Saúde 2015; 12(1):65-69.

40. Nascimento IP. Projeto de vida de adolescentes do ensino médio: um estudo psicossocial sobre suas representações. Imaginario 2006; 12(12):55-80.

41. Bueno CO, Strelhow MRW, Camara SG. Inserção em grupos formais e qualidade de vida entre adolescentes. Psico USF 2010; 15(3):311-320.

42. Becker APS, Maestri TP, Bobato S. T. Impacto da religiosidade na relação entre pais e filhos adolescentes. Arq Bras Psicol 2015; 67(1):84-98.

43. Felipe AOB, Carvalho AMP, Andrade CUB. Espiritualidade e religião como protetores ao uso de drogas em adolescente. SMAD, Rev Eletrônica Saúde Mental Álcool Drog 2015; 11(1):49-58.

44. Del Prette ZAP, Del Prette A. Psicologia das relações interpessoais: Vivências para o trabalho em grupo. Petrópolis: Vozes; 2001.

Artigo apresentado em 14/12/2017

Aprovado em 02/01/2019

Versão final apresentada em 04/01/2019 
